\title{
APOTEOSIS DE LA INFLUENCIA, O DE CÓMO LOS SENDEROS DE LA CIENCIA FICCIÓN HISPANOAMERICANA CONDUCEN A BORGES
}

\author{
POR \\ Luis C. CANO \\ University of Tennessee, Knoxville
}

\begin{abstract}
Si pudiéramos crucificar a Borges lo crucificaríamos
Roberto Bolaño, "Los mitos de Cthulhu"
\end{abstract}

La aserción de Roberto Bolaño (1953-2003), resultado de su desazón por los requerimientos de "amenidad" y "claridad" que, desde su experiencia, han determinado gran parte de la literatura hispanoamericana de los últimos años, brinda variadas posibilidades de lectura. Además de insertarse en un texto que exhibe un tono de consternación por el abandono de los principios que caracterizaron las "grandes literaturas" de mitad del siglo XX y de expresar una reacción de impaciencia por las trayectorias estéticas que se han venido imponiendo en la literatura hispanoamericana, la frase compendia la actitud reverencial de muchos de los autores finiseculares hacia el escritor argentino y su trabajo artístico. En efecto, la figura y producción escritural de Jorge Luis Borges (1899-1986) se han establecido como referentes obligados para múltiples vías de desarrollo de la narrativa hispanoamericana de los últimos 35 años. En contraste con las reacciones negativas experimentadas por los escritores del Boom con posterioridad a los setentas, Borges y su obra no han padecido una reducción en prestigio y admiración. Su figura como personaje histórico, con frecuencia vilipendiada durante su vida por ciertas posiciones políticas, por las críticas y sátiras incisivas a otros autores y obras, por su refinamiento intelectual, por sus volubles opiniones sobre diversos temas o por su desinterés en subscribir a la etiqueta de escritor regional, ha recibido una especie de purificación que lo ha convertido en punto de referencia de las corrientes literarias en años recientes. ${ }^{1}$ Paradigma de todo lo que representan la alta cultura y la

\footnotetext{
1 Aunque sus acotaciones sobre algunos escritores causaron revuelo y, en algunos casos, resentimiento nacionalista ("[Federico García Lorca] me parece un poeta decorativo, un andaluz profesional" (Osorio, 46), fueron algunas de sus perspectivas políticas las que levantaron más intensas oleadas de indignación. En una entrevista concedida a César Hildebrandt, Borges declara: "Yo creo que Pinochet es un buen gobernante. Ese es el único Gobierno posible, así como el de Videla es el único gobierno posible en
} 
literatura de élites en el mundo occidental, sus relatos, paradójicamente, instituyeron condiciones de diálogo con los géneros populares que han surcado con firmeza las corrientes del Boom, el Posboom y ulteriores direcciones en las letras continentales. Este ensayo explora la manera en que la obra y, con mayor frecuencia, la figura de Jorge Luis Borges se han instituido como referente predilecto en los discursos de y sobre la ciencia ficción hispanoamericana a la vuelta del siglo. Además, examina el modo en que esta singularización impacta rasgos temáticos y estilísticos, establece un tipo de recepción asentado en la percepción genérica (escrituralmente hablando) y, finalmente, configura una tradición propia para, al menos, una de las corrientes favorecidas por la ciencia ficción regional.

Para contextualizar esta propuesta, aludiremos a dos procedimientos empleados por los escritores que han incorporado a sus discursos (intra y extra textualmente hablando) indicaciones a Borges y a su producción. El primero de ellos es la inclusión de los "estilemas" con los que se identifica su escritura (en la acepción en la que Umberto Eco utiliza el término). El segundo se estructura a partir de las reiteradas menciones en artículos y entrevistas a su imagen cultural de autor como una manera de establecer una tradición que, simultáneamente, afirma la importancia y problematiza la función de lo popular en las producciones que recurren a modalidades como la ciencia ficción. En línea con los términos empleados por Roland Barthes (1915-1980) cuando indica que "[t]he autor performs a function, the writer an activity" (Critical Essays 144), reflexionaremos en la forma en que los nuevos cultivadores de la Ciencia ficción han retomado al Borges-escritor para la incorporación de los estilemas y al Borges-autor en sus proyecciones culturales.

Comencemos por describir rápidamente el concepto de estilema según lo emplea Eco (1932) en Apocalípticos e integrados (1964). Como parte de su examen de los principios y rasgos constitutivos de la cultura popular, el académico italiano analiza las correlaciones entre los múltiples elementos que conforman el trabajo artístico. El argumento central es que es posible percibir la condición estética del conjunto orgánico en cada uno de los planos de la obra, organizada esta según un "modo de formar" en el que se identifican aspectos de la personalidad del autor y/o las características del período histórico, del contexto socio-cultural y de la escuela en los que se sitúa el texto. La organicidad de la estructura permite el reconocimiento de elementos independientes con rasgos particulares que los relacionan con todos los demás componentes de la estructura mayor. Estos elementos son los que Eco define como estilemas (104). ${ }^{2}$

Argentina. Estoy hablando de determinados países en determinadas épocas. ¿Pero por qué importan tanto mis opiniones políticas?"

2 La noción de estilema ha encontrado un fértil espacio de reflexión en los análisis cinematográficos para determinar las características que establecen el "sello" o "marca" personal de un director. Tales elementos permiten reconocer los estilos de Hitchcock, Truffaut, Kurosawa o Pasolini, por ejemplo. La 
En la historiografía artística, una obra de arte logra establecer un sistema y generar una escuela de seguidores en dos formas. Por un lado, puede proponerse como ejemplo de quehacer estético inspirándose en el cual otros artistas elaboran procedimientos operativos propios y originales o, en la dirección más productiva para este estudio, puede ofrecerle a una generación una serie de estilemas que, incluso, cuando se presentan separados del entorno inicial, evocan sus características y cargan el nuevo contexto con una porción de la aprobación que se le concede a la obra precedente. Así, un trabajo artístico (y, consecuencialmente, su ejecutor) coordinan un sistema de referencias que suscita ciertas reacciones psicológicas en los intérpretes. Como resultado, un texto se experimenta como un sistema de sistemas, algunos de los cuales no apuntan a las relaciones formales internas sino a las conexiones de la obra con los propios receptores y a sus vínculos con el contexto histórico-cultural en que se origina:

A styleme duly "quoted" is not only successful, but confers dignity on its context. It habituates the aesthetic thrill, made inseparable from it now by repetition. For the use of this device, too, there are two possible solutions. First, one can directly evoke a sensation that others have tried and described ... Second, one can introduce already acknowledged commonplaces. (Umberto Eco, citado por Vladiv-Glover 61)

Si bien, en principio, los estilemas se consideraron sólo manifestaciones de tipo estructural, los estudios posestructuralistas mostraron que su funcionalidad trasciende las relaciones sintácticas en la escritura literaria para alcanzar niveles de interacción cultural que sobrepasan los límites del texto. En su ensayo "Biotechnology and Creative Writing," Chung-Hsuan Tung asevera que "[...] when Claude Lèvi-Strauss talks of 'mythemes,' when Umberto Eco talks of 'sememes' and 'stylemes,' and when Frederic Jameson talks of 'ideologemes,' they certainly have in mind the mythical, semiotic, stylistic, and ideological systems". Aunque Tung enfatiza el carácter sistémico y la organicidad de los elementos en la estructura mayor, su comparación entre biotecnología y escritura literaria brinda un enfoque complementario a las implicaciones éticas del trabajo con tales paradigmas. Cerremos estas consideraciones, entonces, explicando la importancia de la noción de estilema para este estudio. En primera instancia, ofrece una especial atención a los roles de autor y receptor y a las negociaciones de sentido que estos establecen, aspecto central en las expectativas que permiten delinear las modalidades/ géneros literarios; segundo, proporciona una serie de ramificaciones relacionadas con

noción ha sido también una herramienta analítica recurrente en la arquitectura (Gaudí, Lloyd Wright), pintura (Dalí, Kahlo), música (Queen, Armstrong), etc. En un nivel más pragmático, la semiótica forense ha desarrollado modelos de trabajo utilizando la estilometría para resolver disputas sobre propiedad intelectual, reconocer autoría o identificar plagios. 
las escuelas artísticas, tradiciones e influencias, relaciones de continuidad entre los escritores y la configuración de la historia literaria; finalmente, reciben una especial importancia al interior de las modalidades populares, materia de particular relevancia en las observaciones que se ofrecen a continuación.

Sin peligro de exageración, es posible afirmar que en la narrativa hispanoamericana del siglo XX no hay otro escritor que más cabalmente haya representado los alcances y el impacto de la noción de estilema que Jorge Luis Borges. De hecho, nuestro uso del concepto no es accidental pues el mismo Eco, como lo expone Deborah Parker en su estudio sobre El nombre de la rosa, "[...] uses Borges in two ways: he exploits both the Argentine writer's work and his cultural image" (843). ${ }^{3}$ Laberintos, espejos, bibliotecas, el/lo otro, el dudoso origen de las fuentes literarias, la supresión de la disociación tiempoeternidad, espacios y tiempos alternativos, hipálages, oximorones y enumeraciones caóticas, el rol de la intertextualidad en la historia literaria, más un largo etcétera, no resultan recursos exclusivos del narrador argentino, pero el modo inconfundible en la que los incorporó a su producción los ha transformado en marcas personales que trascienden los contextos en que se insertan y las circunstancias específicas a las que refieren.

No sobra aclarar, de manera provisional, que, con contadas excepciones, las cuales responden a algún tipo de reescritura de su vida u obra, los escritores y relatos a los que nos estaremos refiriendo, eluden sin reservas la imitación del modelo escritural de Borges. Lo que con mayor frecuencia se observa es una interpretación particular de los rasgos que definen su aproximación estética, aunada a una identificación con la imagen cultural que tanto el escritor mismo como la crítica y los lectores han construido de él como personaje histórico. Sin embargo, el empleo artístico o, en ocasiones, la simple alusión a alguno de los estilemas enumerados, establece automáticamente un vínculo con Borges como fenómeno cultural que, como lo explica Vladiv-Glover, confiere prestigo y, además, conecta con una tradición específica. Cualquier mención a un bibliotecario o un ciego extraviado en laberintos urbanos (José Luis Zárate: “Onarum,” 1999), a la existencia de mundos hipotéticos (Alberto Chimal: Gente de mundo, 1998 o Sergio Meier: La segunda enciclopedia de Tlön,1977), espejos (Emiliano González: "La torre de los espejismos," 1978), discusiones sobre el tiempo y la eternidad (Rodrigo Fresán: El fondo del cielo, 2009) o Gustavo Mosquera: el filme Moebius, 1996), a la literatura y sus fuentes apócrifas (Emiliano González: "La lectura secreta," 1978), la proliferación del individuo (Campo Ricardo Burgos: El clon de Borges, 2010) o la experimentación con variados modelos de escritura popular (la mayor parte de la narrativa de José Luis

\footnotetext{
Umberto Eco utilizó la imagen de Borges, en ambos niveles -intra y extra literario-, en El nombre de la rosa con la inclusión de un bibliotecario ciego con el no particularmente obscuro nombre de Jorge de Burgos. Como acertadamente lo explica Deborah Parker, "What Eco does with Borges is not so much an intertextual move as an incorporation of a cultural image of Borges which is not literary: the appropriation is not so much textual as cultural" (848).
} 
Zárate), suscitan la predisposición a examinar los textos en el marco de las propuestas artísticas del famoso escritor. ${ }^{4}$

Dos precisiones se hacen necesarias. Primero, se da por sobreentendido que similares procedimientos se efectúan con respecto a cualquier artista que ha logrado establecer su marca en la historia literaria, pero la trascendencia excepcional del autor de Ficciones (1944), nuestro interés en la incorporación de las modalidades populares (en especial la ciencia ficción), y la sospecha de que el tratamiento que se haga de ellas en el canon escritural justificaría una re-lectura de los parámetros con que se ha delimitado la periodización literaria hispanoamericana, hacen que Borges constituya un patrón modélico por excelencia. Segundo, el contexto en el que aparecen tales estilemas es esencial para nuestro estudio. Aunque se pueden identificar en múltiples trabajos y modalidades literarias, los que ilustran la propuesta están insertos en un formato ligado a la ciencia ficción y son coherentes con los rasgos que caracterizan tal escritura. También es posible hallarlos en otras manifestaciones narrativas (el relato detectivesco, por ejemplo), con una función análoga, pero con un énfasis mucho más fuerte en los marcadores compositivos.

El segundo procedimiento que un número importante de autores de modalidades populares ha venido ejecutando, y que ha adquirido el valor de estilema por la manera en la que ha sido empleado, es la incorporación del nombre, figura o imagen del escritor argentino en su producción discursiva, sea ésta ficción literaria o cinematográfica, ensayos, textos críticos o en múltiples casos, opiniones y comentarios expresados en medios de difusión mediática a través de blogs y entrevistas. En el famoso ensayo titulado "What is an Author" (1969), Michael Foucault (1926-1984) examina la función del autor como configurador y punto de convergencia de una compleja red de discursos sociales, y argumenta que el nombre del escritor desempeña un papel en la identificación, integración y clasificación de la obra: “[ $\mathrm{t}]$ he author's name manifests the appearance of a certain discursive set and indicates the status of this discourse within a society and a culture" (179). A esto, además, se puede agregar que uno de los procedimientos metonímicos más corrientes es el empleo del nombre del autor para designar su obra y el conjunto de relaciones que la individualizan como trabajo artístico (leí a [Roberto] Bolaño, compré un [Fernando] Botero). En el caso de Borges, en particular, su nombre ha alcanzado proporciones mayúsculas, integrando, además de la producción escritural en los términos delineados antes, su función como intelectual, las interrelaciones estéticas, sus entrevistas y charlas públicas, la omisión de la academia sueca, sus opiniones

4 La construcción de realidades alternativas apoyadas en la obra y la figura de Borges se ha convertido en un productivo espacio para examinar su trabajo y rendirle homenaje. Ver la sección "Ficciones sobre Borges" en Borges múltiple (1999) de Pablo Brescia y Lauro Zavala, o El hacedor. Remake de Agustín Fernández Mallo (2011). Esta última obra fue retirada del mercado a los pocos meses de su publicación por requerimiento de María Kodama, heredera de los derechos de autor de Borges. 
políticas, la conexión teórica con las propuestas estructuralistas y posestructuralistas, su ideología, su erudición; la lista podría extenderse hasta el punto en el que el término "Borges" resulta sinónimo de escritor/autor/intelectual.

Además de tratarse de un autor excepcional cuyo trabajo es un punto de referencia para múltiples discursos críticos y artísticos, ¿qué otras explicaciones podemos proponer para la singularización de Borges en este sistema de referencialidad cultural? Para comenzar, como el escritor argentino y como otros narradores de mitad de siglo que intencionalmente se distanciaron de los parámetros escriturales realistas, una nueva generación de narradores hispanoamericanos se ha enfrentado al cuestionamiento de su hispano/latinoamericanidad. En línea con un confesado interés universalista que enlaza cabalmente con la visión estética de Borges, estos autores rechazan el deber de representar cualquier visión unificada del continente. En los términos de Francisca Noguerol: "Yoss (José Miguel Sánchez), Orlando Vila, Vladimir Hernández, María Elena Durán o Ariel Cruz ... se sienten más cercanos a Borges, Gibson y Tolkien que a la tradición nacional, reclaman como propio un acervo universal" ("Cuentarlo todo. El cuento breve como ejercicio de libertad" 47).

Proclamar a Borges como modelo literario se ha convertido en sinónimo de declarar una poética personal libre, una autonomía artística para elegir el tipo de literatura que se quiera trabajar y el posicionamiento en una tradición específica dentro del marco escritural hispanoamericano. Jorge Volpi (1968), uno de los novelistas que con mayor insistencia ha analizado esta problemática, afirma en El insomnio de Bolivar (2009), refiriéndose a la generación de escritores nacidos en la década de los sesenta:

Hartos de las moralejas oficiales y de los cuentos sobre la identidad nacional, y educados a la sombra de la cultura anglosajona, no tardaron en rebelarse contra el dictado que los obligaba a ser típicamente latinoamericanos. Al lado del grupo mexicano del Crack, el caso más representativo de esta tendencia fue la antología $M c O n d o$, editada por Alberto Fuguet y Sergio Gómez. (46)

La mención de McOndo es particularmente significativa si consideramos que la propuesta que estos autores exponen, según el manifiesto inicial, incluye un inflexible rechazo a cualquier acercamiento que los alinee con el realismo mágico, escritura que con bastante frecuencia, aunque de manera errónea, ha sido identificada con la producción escritural del período del Boom. Además, la reflexión introductoria de la colección rechaza la obsesión por las visiones unificadas de la literatura latinoamericana, impugna el énfasis en la visión ruralizada del continente y valida la importancia de los medios de comunicación de masas en las obras de los escritores incluidos.

Otra razón que ha contribuido a la singularización de Borges como indicador imprescindible es el hecho de que éste no sólo replanteó la propuesta de T. S. Eliot sobre los procedimientos de modificación de las tradiciones literarias (como lo explica 
Patricia Novillo-Corvalán en su ensayo "James Joyce, author of ‘Funes el memorioso"”), sino que en sus propios escritos insistentemente delineó posibles configuraciones para la disposición de su obra en la historia literaria occidental. En particular, Borges fue especialmente insistente en incluir alusiones a escritores cuyo trabajo permitió sentar las bases para el desarrollo de la ciencia ficción en el mundo occidental. Su interés por la modalidad aparece reiterado en las entusiastas alusiones a los relatos de H. G. Wells, Ray Bradbury, Leopoldo Lugones, Olaf Stapledon y Adolfo Bioy Casares, incluidos en prólogos y notas para varias publicaciones. Es muy frecuente, por ejemplo, que en sus observaciones a la obra de otros escritores, incluya un inserto con referencia a la importancia de la cuentística de Poe en la conformación de las narraciones detectivescas y la ciencia ficción. ${ }^{5}$

Un procedimiento afín ha sido empleado por los nuevos escritores cuando aluden a la obra de Borges. En ensayos, artículos, ponencias, antologías, comentarios en blogs, foros, redes sociales y entrevistas aparecen incontables menciones a la influencia que el escritor ejerció en su formación literaria, recurso que encaja adecuadamente con la propuesta (ficcional y no ficcional) del argentino. El chileno Jorge Baradit (1969), autor de Ygdrasil (2005), Synco (2008) y Policía del karma (2011), entre otras, evoca el impacto que le produjo la lectura de un cuento de Borges:

[...] por un accidente a los 11 años ... [un] tío me prestó un alto de Sandokanes, John Carters, Julios Vernes y entremedio un libro que contenía, como un parásito escondido al fondo de un sandwich, "La Lotería en Babilonia"...

Ese cuento obsceno parásito de Borges que se me enquistó en la cabeza, funciona perfecto como una metáfora de la desesperada búsqueda de sentido en medio del vacío ...

"La Lotería en Babilonia" se me hace un ejercicio maravilloso de construir mundo a partir de una premisa absurda que crece y crece cancerígena apoyándose en sus constructos previos, estirando ramas como enredadera carnívora ... Me conmueve este cuento, me encantan los bestiarios. ("Un parásito")

Y el mexicano Alberto Chimal (1970), uno de los escritores de ciencia ficción que con más entusiasmo ha declarado su deuda con Borges, escribe:

en uno de los números de la revista [Ciencia y Desarrollo], hallé "Tlön, Uqbar, Orbis Tertius" ... Debo haberme sorprendido, tal vez, de ver [a Borges] presentado como escritor de ciencia ficción, pues también suponía que ese tipo de historias no eran escritas por autores de lengua española ... mi historia con Tlön y Uqbar y Orbis Tertius tiene todo que ver con mis emociones. Qué inquietante encontrar una

\footnotetext{
5 Ver, por ejemplo, "John Dickson Carr. It Walks by Night," "Wilkie Collins. La piedra lunar," "Sobre
} Chesterton" o "Cuentos argentinos," recopilados en Obra crítica. Volumen II. 
narración donde el problema - un mundo que se inserta en éste, que lo abruma y lo conquista y al final lo borra- no se resuelve en absoluto. Qué escandaloso asomarse (sic) la filosofía y la ciencia de Tlön y notar que no defienden el racionalismo y más bien se acercan al idealismo, a los modelos delirantes de los filósofos idealistas, y qué abrumador ver que todos en el cuento empiezan a creer que el mundo es así: que los objetos no siempre existen y que el acto de contar modifica la cantidad que se cuenta. Qué abrumador también darse cuenta de que una historia arbitraria puede modificar la percepción del mundo, la conciencia misma del mundo, y luego entender que Borges, al mostrar este triunfo de lo subjetivo, no reafirma la realidad del universo, sino que la demuele, la destruye sistemáticamente hasta que ya no quedan asideros ni tranquilidad posibles... la lectura de ese cuento en esa revista en ese momento de la vida me convenció del poder infinito de la imaginación, y también de que la literatura podía ser mucho más: más que cualquier historia que hubiera leído hasta aquel momento. Ya inventaba cuentos y ya quería ser escritor, pero entonces lo supe con claridad, como nunca antes. Y entonces entendí, también clarísimamente, que iba a ser muy difícil: que cada obra hecha posible por un gran autor es una inspiración y un estímulo, pero también un desafío. Y que nada iba a tener sentido si el escribir no ofrecía algún estremecimiento como el que yo había experimentado: si no daba algún recordatorio de lo humano en el mundo o por lo menos de lo humano en el lector. Ya he dicho que Borges es inagotable: he leído su obra entera, he leído su obra en colaboración, he visitado a sus biógrafos y sus amigos, y en el mapa del mundo he ido hasta su casa. ("Una presencia de Borges")

Las reminiscencias de Chimal no sólo destacan la poderosa huella intelectual y la conmoción estética producida por la experiencia de lectura sino que, más significativo para nuestras reflexiones, exaltan la importancia del impacto emotivo en el acto de recepción en una forma que recupera la línea reflexiva asumida por Borges en sus comentarios sobre The Invisible Man o The Martian Chronicles. ${ }^{6}$ La promoción de la imagen y la obra del escritor, inserta en la estrategia de identificación de precursores, trasciende el nivel de reconocimiento de influencias para ejercer un impacto en la lectura y relectura de la producción narrativa de Borges, en la validación de sus propuestas estéticas y, adicionalmente, en la constitución de una tradición propia a la cual se afilian, componentes fundamentales en la percepción e impulso de una modalidad cuyo prestigio aún padece los efectos de una larga historia de desaprobación crítica.

6 La lista de escritores que en años recientes han reconocido con admiración -en ocasiones de manera ferviente- la influencia de Borges incluye un catálogo de los nombres más reconocidos vinculados a la Ciencia ficción continental: Roberto Bolaño, Carmen Boullosa (1954), Campo Ricardo Burgos (1966), Carlos Chernov (1953), Michel Encinosa Fu (1974), Rodolfo Fogwill (1941-2010), Rodrigo Fresán (1963), Emiliano González (1955), Álvaro Menéndez Leal (Menen Desleal, 1931-2000), Sergio Meier (1965-2009), Gustavo Nielsen (1962), Ignacio Padilla (1968), Alan Pauls (1959), Edmundo Paz Soldán (1967), Enrique Prochazka (1960), José Miguel Sánchez Gómez (Yoss, 1969), Ana Solari (1957), José Luis Zárate (1966), Mike Wilson (1974), entre los más destacados por lectores y críticos. 
Ciertamente, el recurso descrito no es una innovación de los escritores hispanoamericanos contemporáneos. El interés por las modalidades artísticas designadas con el adjetivo "popular" ha alcanzado niveles inéditos como extensión de la percepción cada vez más generalizada de "pop culture as the 'default' form of culture" (Danesi vii) que se ha venido afirmando en el siglo XXI. La ciencia ficción, la narrativa detectivesca, las diversas variaciones de lo fantástico, el horror, el new weird, romance, erotismo, etc., no sólo dominan las listas de Best Sellers internacionales, sino que su éxito creciente ha inducido una redefinición de lo que es "culto" o "popular". No sorprende, entonces, que el énfasis en la adopción de escrituras genéricas haya ido acompañado por la exploración de sus principios constitutivos y por una revisión crítica de los autores y movimientos que se proponen como antecedentes directos. Un resultado de este proceso revisionista es la exploración retrospectiva y la renovación de una amplia red de discursos que florecieron a mediados del siglo XX. Los análisis narratológicos, la disección estructural, las teorías de la recepción, direcciones analíticas que, aunque no totalmente ausentes de las investigaciones literarias contemporáneas, habían languidecido en un campo abrumadoramente dominado por los estudios culturales se han revitalizado centrando su atención en los estudios genéricos (genre studies). Y, para Hispanoamérica, el punto de referencia más prestigioso (aunque no el único o el más prolífico en cuanto a producción) es Jorge Luis Borges. Esto explica la profusión de charlas y estudios académicos sobre el autor y su obra ofrecidos por los escritores, ${ }^{7}$ el cada vez mayor número de obras literarias que lo incluyen como personaje, la aparición de publicaciones que le rinden homenaje (muchas de ellas apuntaladas en la celebración del centenario de su nacimiento) y, como también es de esperarse, las que optan por enfatizar una perspectiva intencionalmente irreverente que rechaza la idolización/idealización de la que ha sido objeto. ${ }^{8}$

En esta instancia nos encontramos, entonces, ante el tercer argumento para la particularización de Borges como componente del proyecto de los escritores de ciencia ficción en Hispanoamérica en los últimos años: la trascendencia asignada a las modalidades populares como punto de partida de sus programas escriturales. En su mayor parte, los narradores que han mostrado interés en demarcar una línea de continuidad con Borges y su obra lo han hecho como parte de proyectos que operan bajo los principios constitutivos de las narrativas de género. En particular, el relato detectivesco y la ciencia ficción se han constituido como los terrenos más fecundos para configurar tales conexiones. Aunque, en principio, parecería difícil establecer una nexo entre las intelectualizadas ejecuciones de Borges y las aproximaciones más convencionales de los escritores a los que nos hemos estado mencionando, las relaciones no sólo aparecen en el nivel

Guillermo Martínez, Alan Pauls, Alberto Chimal y Roberto Bolaño son algunos de los autores que se han dedicado al análisis académico de la obra de Borges.

8 En 1978 Juan Fló compiló una serie de artículos en el libro titulado Contra Borges, y en 1999, para el centenario de su nacimiento, Martín Lafforgue publicó la colección de textos titulada Antiborges. 
superficial de las obras a través de llamadas de atención intratextuales directas como en Cárcel de árboles (1991) de Rodrigo Rey Rosa (1958) o "El derby de los penúltimos" (1998) de Fernando Iwasaki (1961), sino que también afloran en espacios no ficcionales como sus investigaciones académicas (Pauls, Chimal), evaluaciones críticas (Bolaño, Martínez), o en comentarios y opiniones de tipo personal (Yoss, Padilla). Dos inquietudes se desprenden de las afirmaciones previas. Primero, cómo se explica y en qué dirección se canaliza el interés de Borges por estas modalidades y, en segunda instancia, qué ha motivado a los nuevos narradores que incorporan en sus trabajos estas vertientes escriturales, a establecer como referente la obra del argentino.

Una constante fundamental en la composición de los géneros populares es la necesidad de balancear la transgresión de los principios de verosimilitud realista adoptando formatos narrativos más tradicionales, rasgo que tanto el relato detectivesco como la ciencia ficción acentúan. Sin embargo, mientras múltiples variedades del primero exhiben estructuras rígidas acompañadas de fórmulas previsibles, como ya lo han analizaron Tzvetan Todorov, John Cawelti y el mismo Eco, ${ }^{9}$ la ciencia ficción en general y, muy especialmente la producida en Hispanoamérica, muestra escenarios diferentes. En contraste con la rigidez estructural que ha sido ostensible en la narrativa detectivesca, la mayor parte de la ciencia ficción hispanoamericana favorece estructuras flexibles optando, más bien, por la inclusión de leitmotivs identificados con la modalidad. Individuos obsesionados por el conocimiento, formulación de realidades alternativas y universos paralelos, anomalías temporales y desplazamientos espaciales, son algunos de los motivos tradicionales que aparecen en la obra de los autores hispanoamericanos a lo largo del siglo XX (incluyendo a Borges) y que continúan con una presencia destacada en la ciencia ficción de los últimos 35 años.

Por otro lado, es importante recordar que Borges hizo contante hincapié en la exaltación de la peripecia sobre las motivaciones psicológicas en la narración literaria, algo que exaltó de forma repetida en múltiples comentarios sobre las modalidades populares. En el prólogo a la Invención de Morel (1940) de Adolfo Bioy Casares (19141999), Borges enumera una lista de novelas del siglo XX que califica como poseedoras de grandes argumentos: "[...] considero que ninguna otra época posee novelas de tan admirable argumento como The Invisible Man, como The Turn of the Screw, como Der Prozess, como Le Voyageur sur la terre, como ésta que ha logrado, en Buenos Aires, Adolfo Bioy Casares" (vi). No es casualidad que de las cinco obras mencionadas una sea un modelo clásico de la literatura fantástica (la de Henry James) y dos sean ejemplos

9 Para ampliar la información sobre la composición del relato detectivesco referirse a John G Cawelti, Adventure, Mystery, and Romance: Formula Stories as Art and Popular Culture (Chicago y Londres: U of Chicago P, 1976); Umberto Eco y Thomas A. Sebeok, Eds. The Sign of the Three (Bloomington e Indianapolis: Indiana UP, 1988. Tzvetan Todorov, "The Typology of Detective Fiction." The Poetics of the Prose. Trad. Richard Howard, (Ithaca: Cornell UP, 1977. 42-52). 
de ciencia ficción (las de Wells y Bioy Casares), escrituras que, en la opinión de Borges, despliegan en el nivel más superficial el carácter artificial de lo literario.

Un rasgo singular del acercamiento de Borges a tales modalidades es que, a pesar de haberse efectuado desde una posición marcadamente intelectual, no muestra el distanciamiento irónico o el tono paródico empleado por muchos escritores del Boom y el Posboom, tal es el caso de Julio Cortázar, Gabriel García Márquez, Severo Sarduy y Ana Lydia Vega, entre otros. De hecho, una de las críticas que Stanislaw Lem (19212006) le hace en el artículo "Unitas Oppositorum: The Prose of Jorge Luis Borges" (1984), es precisamente lo que Lem considera como una falta de compromiso con el material que Borges selecciona como base de sus escritos: "Borges is successful because in any single case he never questions the implied premises of the model structure that he transforms" (236).

Si bien Lem acierta en su sospecha de que Borges se halla más interesado en la disección de las estructuras y el examen de los elementos constitutivos que en el análisis de la repercusión social o el impacto que la ciencia ficción haya podido tener en el diseño de la historiografía literaria, esta observación no puede generalizarse para todas las modalidades con las que trabajó. En uno de los muchos elogios que escribió sobre las contribuciones de Poe al desarrollo del relato detectivesco, Borges ofrece una clave para entender su acercamiento a las escrituras populares:

In 1840 Edgar Allan Poe enriched literature with a new genre. This genre is above all ingenious and artificial, real crimes are not commonly discovered by abstract reasoning but by chance, investigation, or confession. Poe invented the first detective in literature ... He invented at the same time the convention, latter classical, that the exploits of the hero should be told by an admiring and mediocre friend. (citado por Maurice Bennett, 264)

El acento en el efecto intelectual, unido a la atención conferida al convencionalismo y la artificialidad argumental del relato detectivesco, contrasta con la emotiva reacción a la lectura de The Martian Chronicles y The Time Machine. En el prólogo que preparó para la primera edición en español de la novela de Ray Bradbury (1920-2012), un inusitadamente emotivo Borges exclama: “¿Qué ha hecho este hombre de Illinois, me pregunto, al cerrar las páginas de su libro, para que episodios de la conquista de otro planeta me pueblen de terror y de soledad? ¿Cómo pueden tocarme estas fantasías, $y$ de una manera tan íntima?" (Obra crítica. Volumen 2). Y en el texto introductorio a Charles Howard Hinton. Relatos cientificos, califica The Time Machine como una "admirable pesadilla" (Obra crítica. Volumen 2). La repetida llamada de atención sobre el impacto emocional, aunada al ya señalado interés en la composición textual, ilustran la postura de Borges hacia esta modalidad: curiosidad intelectual y emoción receptiva se integran para delinear sus proyectos estéticos relacionados con la ciencia ficción. Estos 
atributos, evidentemente, han sido advertidos por otros narradores, como lo demuestran los escritos de Chimal y Baradit citados en páginas anteriores.

Por otro lado, su interés por racionalizar los procedimientos empleados en las modalidades populares le permitió intuir, con considerable precisión, los tres rasgos más insistentemente asociados a la ciencia ficción: disociación de la dimensiones temporales intra y extraliteraria, acogida de un discurso que reconstruye, imita o parodia los rasgos tradicionalmente aceptados como propios de la escritura científica, y la firme actitud crítica que sondea el desarrollo de nuevas tecnologías, examina la forma en que estas han sido manipuladas, y formula juicios sobre el papel del científico en su sociedad. ${ }^{10} \mathrm{De}$ no menor importancia es que Borges no solamente dirigió su atención a las influencias foráneas que influyeron en las visiones artísticas y en el desarrollo de la ciencia ficción en Hispanoamérica (mayormente escritores de Inglaterra y EE.UU.), sino que sus conclusiones se desprenden de la lectura de uno de los más importantes alentadores de la ciencia ficción continental: Leopoldo Lugones.

En el prólogo a Cuentos argentinos, parte de la colección Biblioteca de Babel, Borges expresa un respetuoso aprecio por la destreza en que Las fuerzas extrañas (1906) armoniza las mejores contribuciones del Modernismo con las influencias de Poe y Wells. Su celebración de "Yzur," el cual considera el más logrado de los relatos de la colección, encapsula los tres rasgos descritos (investigación, estilo, temporalidad) $\mathrm{y}$, además, anuncia las variantes de recepción y la proximidad que la modalidad puede tener con el relato fantástico:

[...] El argumento de Lugones exigía que su narrador fuera un hombre de ciencia, hecho que debemos agradecer, ya que le impuso un estilo severo. [...] La historia es singular; para no delatar su contenido, sólo la juzgaremos a grandes rasgos. Puede ser leída de dos maneras. La primera sería considerarla la narración de un experimento extraordinario; la segunda es la crónica de dos seres que, a lo largo del tiempo, se enloquecen y de algún modo amalgaman la bestialidad y la humanidad. La página final puede ser realista, pero asimismo puede ser alucinatoria. (Obra crítica Volumen 2)

Las observaciones previas nos permiten reconocer una diferencia fundamental en la forma en la que Borges se acercó al relato de detectives y a la ciencia ficción. En ambos casos, es evidente un acentuado interés por decodificar los marcadores que las definen y delimitan, en particular las operaciones compositivas que permiten configurarlas como modalidades. No obstante, mientras en el caso de la narración detectivesca la atención minuciosa a procedimientos y estructuras comporta una experiencia predominantemente intelectual, la ciencia ficción matiza el componente estructural suscitando un impacto

${ }_{10}$ Para ampliación de este tema referirse a mi estudio Intermitente recurrencia incluido en la bibliografía. 
emotivo en la experiencia de recepción. El énfasis en una u otra dirección explicaría, también, la inclusión de formatos y estilemas claramente discernibles en escritores como Iwasaki, Guillermo Martínez (1962) o Pablo de Santis (1963), mientras que los autores de ciencia ficción que reconocen la influencia de la producción narrativa del argentino enfatizan los efectos sobre el rigor en la aplicación de los proyectos formales.

Se podría argüir que, a nivel conceptual, los relatos de ciencia ficción de Borges son tan sofisticados intelectualmente como los detectivescos y que, además, es común que en su obra las dos modalidades se entretejan como ocurre en "El jardín de senderos que se bifurcan" (1941) y, con más sutileza en "Tlön, Uqbar, Orbis Tertius" (1940), ambas incluidas en Ficciones (1944). Sin embargo, también es cierto que estos cuentos (al igual que "La Biblioteca de Babel" (1941) y "La Lotería en Babilonia" (1941), otros dos relatos frecuentemente leídos como manifestaciones de la ciencia ficción en la escritura de Borges) expresan y promueven reacciones emotivas que no se destacan en, por ejemplo, "La muerte y la brújula". En uno de los pasajes de "Tlön" leemos:

Me puse a hojear [el libro] y sentí un vértigo asombrado y ligero que no describiré, porque ésta no es la historia de mis emociones sino de Uqbar y Tlön y Orbis Tertius. En una noche del Islam que se llama la Noche de las Noches se abren de par en par las secretas puertas del cielo y es más dulce el agua en los cántaros; si esas puertas se abrieran, no sentiría lo que en esa tarde sentí. (Prosa completa. Volumen 1 318-19)

Una actitud similar podemos detectar en las obras de los escritores hispanoamericanos de ciencia ficción de años recientes, los cuales proyectan una ausencia notable de distanciamiento irónico o paródico en sus trabajos, en agudo contraste con la forma en la que narradores de ciencia ficción de los sesentas y setentas como Angélica Gorodischer (1928), Eduardo Goligorsky (1931) y Alberto Vanasco (1925-1993), encauzaron su interés por la modalidad. Esta empatía estética con el enfoque de Borges resulta especialmente significativa si consideramos que la mayoría de estos narradores se formaron intelectual y artísticamente bajo las estrategias ideológicas y discursivas del epistema posmoderno, determinado por convenciones literarias que se canalizan en discursos paródicos autorreflexivos. Con contadas excepciones, entre ellas la novela Xanto, Novelucha libre (1994) o el cuento "El viajero" (1992), obras del mexicano José Luis Zárate, o el comic Perramus, la isla del guano, una fábula grotesca y desmesurada en la que Jorge Luis Borges, el gran olvidado de la Academia, obtiene finalmente el Premio Nobel (1993) de Juan Sasturain (1945) y Alberto Breccia (1919-1993), la mayor parte de la ciencia ficción escrita con posterioridad a 1980 prescinde de las formulaciones paródicas o los distanciamientos irónicos, prácticas omnipresentes en la narrativa posmoderna, optando en su lugar por un retorno al empleo de la modalidad como vehículo de evaluación crítica de los referentes extratextuales que constituyen el punto de partida de los relatos. Situados en un momento de transición que comporta cambios en las exigencias culturales 
relacionadas con su papel como intelectuales en Hispanoamérica, las presiones por una literatura más accesible impuestas por la industria editorial, y la influencia omnipresente de los medios de comunicación, los escritores de ciencia ficción de décadas recientes postulan una salida a la ironía y parodia autorreferenciales favoreciendo un retorno a varios de los principios retóricos y temáticos que definieron la Ciencia ficción continental previa a la aparición del Boom latinoamericano. Las distopías posapocalípticas (Anatomía humana, 1993, de Carlos Chernov; Zack, 1993, de Ana Solari), la exploración de universos paralelos (Fábulas de una abuela extraterrestre de Daína Chaviano, 2003) o de realidades alternativas ("Recuerdos de un país zombi" del cubano Erick Mota, 2013), las cada vez más populares ucronías (La Habana Underguater de Erick Mota, 2010; el proyecto dirigido por Jorge Baradit et al CHILA: Relación del Reyno (1495-2210), 2010), no sólo eluden el tipo de transpolación futurista con el que más frecuentemente se asocia la CF, sino que muchas de ellas incluyen como motivo una romántica búsqueda de núcleos de sentido apuntalados en diferentes instancias del pasado. En este escenario adquieren sentido los procedimientos que hemos venido identificando a lo largo de este estudio: la adopción de estilemas que remiten a la obra de una de las voces más prestigiosas de mitad del siglo anterior, el interés en diseccionar los modelos o identificar los momentos cumbres del reconocimiento de la Ciencia ficción continental, la validación de la visión menos escéptica de las narrativas populares y, como una conjunción de todo lo anterior, la figura de Borges. De hecho, un argumento análogo podría hacerse extensivo a la tendencia cyberpunk, otra dirección crecientemente popular entre los nuevos narradores de la Ciencia ficción hispanoamericana, con la puesta en narrativa del dramático contraste entre un futuro inmediato tecnológicamente avanzado y el tono de desilusión, disconformidad o rechazo concretados en un héroe con ideas y principios firmemente enraizados en una visión retrospectiva de tipo romántico. ${ }^{11}$

El procedimiento no es desemejante a lo que el mismo Borges predicó como parte de su disposición artística e, incluso, de su visión del mundo. En la imagen pública que desplegó con posterioridad a su conflicto con el peronismo, el argentino puso gran vehemencia en señalar un distanciamiento con el mundo contemporáneo, exaltando en su lugar la importancia de otras eras, no necesariamente ancladas en marcadores históricos específicos. Sus entrevistas y conferencias enfatizan tal razonamiento:

El presente es siempre atroz... Nadie se siente feliz en el presente. La felicidad corresponde más bien al pasado, a la nostalgia, a la esperanza. En otras épocas la gente no tenía conciencia histórica del tiempo en que estaba viviendo. En cambio ahora, estamos pensando constantemente en el momento histórico que vivimos y eso no nos hace ni muy sabios, ni muy felices. (El historiador)

${ }^{11}$ Para una descripción detallada del cyberpunk referirse a la obra de Sabine Heuser, Virtual Geographies. Cyberpunk at the Intersection of the Postmodern and Science Fiction. 
También su obra es coherente con esta inclinación a remover marcas temporales inscritas en el presente eligiendo, en su lugar, una exploración de referentes discursivos removidos de contextualidad histórica y social. Varios comentaristas de la obra de Borges señalaron esta preferencia atribuida al pasado en desmedro del presente (Lem, V. S. Naipaul) o del futuro (Ilan Stavans). En “Unitas Oppositorum” un escéptico Lem escribe:

[Borges] has lit up -given a paradoxical resurrection to- the treasures transmitted to us from the past. But he will not succeed in keeping them alive for any long period of time. Not because he has a second-rate mind, but because, I believe, such a resurrection of transitory things is in our time quite impossible. His work, admirable though it may be, is located in its entirety at an opposite pole from the direction of our fate. Even this great master of the logically immaculate paradox cannot "alloy" our world's fate with his own work. (241-242)

Tanto en el trabajo deliberadamente literario como en sus prólogos, comentarios y entrevistas, Borges ilustra en la práctica la base conceptual que han seguido las teorías de géneros a partir de la inclusión del concepto de intertextualidad. En "Generic Identity and Intetextuality," Marko Juvan afirma la importancia de la intertextualidad como criterio útil para la determinación de configuraciones genéricas (genres). En su propuesta, Juvan explica cómo los géneros existen y funcionan en la medida en que se hallan insertos en prácticas sociales que demarcan conexiones inter y metatextuales con otros textos ("Genres live on social practices which frame intertextual and metatextual references to prototypical texts or sets of texts" 10). Como resultado, la historia literaria no sólo determina los antecedentes genéricos de un trabajo (a posteriori, debemos agregar), sino que los artistas mismos, conscientes de sus propios proyectos, y la industria de producción y distribución, consciente de sus motivaciones e intereses, emplean estrategias que ponen en juego todo un perfil semántico y estético en el que debe efectuarse la lectura del texto, siempre con respecto a los parámetros de la modalidad específica en la que se inserta (Juvan 8). Las prácticas descritas modelan las pautas de recepción de potenciales lectores ya educados/entrenados, sea por la academia o por sus propias lecturas, en el reconocimiento de las constantes que constituyen un género.

Ala luz de las observaciones previas, el conjunto de la producción de Borges aparece como un proyecto coherente de exploración de principios compositivos, de constante identificación de fuentes e influencias, y de reconocimiento de la manera como estas se insertan en su proyecto estético, prácticas que los escritores de años recientes han adoptado tomando como modelo la obra y la figura del autor. Para los escritores interesados en incorporar las modalidades populares en su producción y más específicamente para los que trabajan con la ciencia ficción, este recurso desempeña varias funciones. En primer lugar, constituye un elemento cohesionador en un período caracterizado por el rechazo a las nociones de latino/hispanoamericanidad empleadas como rótulos homogeneizadores. 
No importa el área cultural o la generación en la que se sitúen, el reconocimiento de la influencia de Borges ofrece un vínculo de unión en un ambiente de dispersión temática, estilística y generacional. En segunda instancia, proporciona un referente respetable para el trabajo que se viene haciendo con las modalidades populares. En efecto, cada escritor que opta por el tratamiento de estas recibe una validación retrospectiva cuando afirma la influencia del argentino. No importa si el nexo se establece a través de un imaginario que superpone las experiencias individuales a los supuestos culturales, el resultado es el mismo: concreta un linaje prestigioso que permite manejar el desconcierto sobre el lugar que ocupan en la producción artística del continente y afirma su posición en la historia literaria. Lo anterior, adicionalmente, configura un marco para la constitución de una tradición específica (trátese de la literatura fantástica, la narrativa detectivesca o la ciencia ficción) la cual se apuntala con orgullo en referentes locales.

Los alcances de esta visión en el marco de la ciencia ficción han sido agudamente representados por el colombiano Campo Ricardo Burgos López en su novela El clon de Borges (2011). En la obra, uno de los personajes que, sin ningún escrúpulo, acepta padecer de "Borgeslatría," fabrica un clon del autor el cual conserva intacta la conciencia del individuo original y, más importante aún, continúa acrecentando la admirable obra de su antecesor. La potencial reproducción ad aeternum del escritor no sólo pone en juego todos los dilemas éticos a que se ha visto sujeto el procedimiento de clonación sino que, además, amenaza con rediseñar todo el entramado de la literatura occidental con la redefinición de conceptos como "originalidad", "influencias", "antecedentes literarios" y "géneros". En cierto momento, otro de los personajes imagina un escenario utópico en el que un mismo escritor a lo largo de vidas consecutivas podría llegar a constituir un género literario compuesto exclusivamente por sus escritos.

Más allá de si Jorge Luis Borges y su obra detentan o no esa caja negra en la que la utopía se transforma en ciencia ficción o la ciencia ficción en utopía, como postula Csicsery-Ronay ("Notes on Mutopia") y como artísticamente concreta Burgos en su novela, la función que Borges ostenta como indicador cultural y principio integrador para el entretejimiento de las narrativas populares con los discursos del canon a la vuelta de siglo es incuestionable. El reconocimiento de esta particularidad va más allá de simplemente avalar la existencia de una tradición propia para la ciencia ficción hispanoamericana; más significativo aún, afirma la viabilidad de una relectura de la historiografía literaria continental centrada en las intersecciones entre lo popular y lo canónico.

\section{BiBLIOGRAFÍA}

Baradit, Jorge y otros. CHILZ: Relación del Reyno (1495-2210). Ediciones B, 2010. "Un parásito escondido al fondo de un sándwich". 60 Watts. 29 marzo 2013. $<60$ watts.cl/2013/03/mi-cuento-favorito-jorge-bradit/>. 21 enero 2014. 
Barthes, Roland. "Authors and Writers". Critical Essays. Richard Howard, ed. Evanston: Northwestern UP, 1972. 143-150

Bennett, Maurice J. "The Detective Fiction of Poe and Borges." Comparative Literature 35/3 (1985): 262-275

Borges, Jorge Luis. Obra crítica. Volumen II. Librodot.com. 7 nov. 2013. "Prólogo". Bioy Casares, Adolfo. La invención de Morel. New York: Penguin, 1996. v-vii.

Prosa completa. Volumen 1. Barcelona: Bruguera, 1980.

Breccia, Alberto y Juan Sasturain. Perramus, la isla del guano. Ediciones B: 1993. Brescia, Pablo y Lauro Zavala, eds. Borges múltiple. México: El Estudio, 1999. Burgos López, Campo Ricardo. El clon de Borges. Bogota: Edición de autor, 2010. Cano, Luis. Intermitente rrecurrencia: la ciencia ficción y el canon literario en Hispanoamérica. Buenos Aires: Corregidor, 2006.

Chaviano, Daína. Fábulas de una abuela extraterrestre. La Habana: Editorial Letras Cubanas, 1988.

Chernov, Carlos. Anatomía humana. Buenos Aires: Planeta, 1993.

Chimal, Alberto. "Una presencia de Borges". Las historias. 6/8/2012. Web. Octubre 14, 2013.

Csicsery-Ronay, Istvan. "Notes on Mutopia”. Página del autor, 1997. Web. Enero 31, 2014.

Danesi, Marcel. Popular Culture. Introductory Perspectives. Lanham: Rowman and Littlefield, 2008.

Eco, Umberto. Apocalípticos e integrados. Andrés Boglar, trad. Barcelona: Lumen, 1981.

El historiador. "Entrevista a Jorge Luis Borges". Web. Octubre 12, 2013.

Fernández Mallo, Agustín. El hacedor (de Borges), Remake. Madrid: Alfaguara, 2011. Fló, Juan, ed. Contra Borges. Buenos Aires: Galerna, 1978.

Foucault, Michael. "What is an Author". Modern Criticism and Theory. A Reader. David Lodge y Nigel Wood, eds. Joseph V. Harari, trad. Singapore: Longman, 2000. 173-187.

Fuguet, Alberto y Sergio Gómez, eds. "Presentación del País McOndo". McOndo. Barcelona: Grijalbo-Mondadori, 1996.

González, Emiliano. "La lectura secreta". Los sueños de la bella durmiente. Tabasco: Editorial Joaquín Morliz, 1987. 44. Web 12 diciembre. 2013.

"La torre de los espejismos". Los sueños de la bella durmiente. Tabasco: Editorial Juaquín Morliz, 1978. 87. Web. 12 dic. 2013.

Heuser, Sabine. Virtual Geographies. Cyberpunk at the Intersection of the Postmodern and Science Fiction. Nueva York: Rodopi, 2003.

Hildebrandt, César. "Jorge Luis Borges”. A qué palo te arrimas. <estoespurocuento. wordpress.com>. 05/14/2013. 8 dic. 2013. 
Iwasaki, Fernando. "El derby de los penúltimos". Renacimiento 25/26 (1999): 60-70. Juvan, Marko. "Generic Identity and Intertextuality". CLCWeb: Comparative Literature and Culture 7/1 (2005). 12 enero 2014.

Lafforgue, Martín, ed. Antiborges. Barcelona: Javier Vergara, 1999.

Lem, Stanislaw. "Unitas Oppositorum: The Prose of Jorge Luis Borges". Microworlds:

Writings on Science Fiction and Fantasy. Franz Rottensteiner, trad. San Diego: Harcourt Brace Jovanovich, 1984. 233-242.

McLaughlin, Robert L. "Post-Postmodern Discontent: Contemporary Fiction and the Social World". Symplokē 12-1/2 (2004): 53-68.

Mota, Erick J. La Habana Underguater. Atom Press, 2010.

"Recuerdos de un país zombi". Terra Nova. Antología de ciencia ficción contemporánea. Villarreal, Mariano y Luis Pestarini. Sportula, 2012. 57-108.

Naipaul, V.S. "Comprehending Borges." The New York Times Review of Books 10/19/1972. Enero 16, 2014.

Noguerol, Francisca. "Cuentarlo todo. El cuento breve como ejercicio de libertad". El cuento hispanoamericano contemporáneo. Vivir del cuento. Adélaïde de Chatellus, ed. Sarreguemines: Rilma2-ADEHL, 2009.

Novillo-Corvalán, Patricia. "James Joyce, Author of "Funes el memorioso". Variaciones Borges 26 (2010): 59-82. Enero 25, 2014.

Osorio, Jairo y Carlos Bueno, eds. Borges. Memoria de un gesto. Medellín: Alcaldía de Medellín, 1979.

Parker, Deborah. "The Literature of Appropriation: Eco's Use of Borges in Il nome della rosa". The Modern Language Review 85/4 (1990): 842-849.

Rey Rosa, Rodrigo. The Pelcari Project. Cárcel de árboles. Paul Bowles, trad. Tiburón: Cadmus, 1997.

Stavans, Ilan. "Borges and the Future." Science Fiction Studies 17/1 (1990): 77-83.

Vladiv-Glover, Slobodanka. "The Poetics of Pastiche in Eco's Postmodern Detective Novel”. European Legacy 13/1 (2008): 59-81. Academic Search Complete. Web. 17 Diciembre, 2013.

Solari, Ana. Zack. Montevideo: Trilce, 1993.

Tung, Chung-hsuan. “Biotechnology and Creative Writing.”. 4 enero 2014.

Volpi, Jorge. El insomnio de Bolivvar. México: Random House-Mondadori, 2010.

Zárate, José Luis. "Onarum". Quitzä y otros sitios. Puebla: Secretaría de Cultura, 2002. 39-42.

"El viajero". Axxón 160 (2006). 4 enero 2012. Xanto. Novelucha Libre. México: Planeta, 1994. 